\title{
OsteoConduct: Wireless Body-Area Communication based on Bone Conduction
}

\author{
Lin Zhong $^{1} \quad$ Dania El-Daye $^{2} \quad$ Brett Kaufman $^{1} \quad$ Nick Tobaoda $^{2} \quad$ Tamer Mohamed $^{1} \quad$ Michael Liebschner $^{2}$ \\ ${ }^{1}$ Dept. of Electrical \& Computer Engineering \\ ${ }^{2}$ Dept. of Bioengineering \\ Rice University \\ 6100 Main St, Houston, TX 77005
}

\begin{abstract}
We present OsteoConduct, a novel technology that leverages the human musculoskeletal system to transmit data and interface users in a low-power, secure, non-intrusive fashion. OsteoConduct employs a mechanical stimulus in form of patterned acoustic vibration, generated by human users or external stimulators, and a low-cost receiver, as simple as an accelerometer or microphone. It is particularly suitable for low data rate communication between implantable or wearable devices, especially as a secure and lowpower alternative to wireless body-area network technologies, such as Bluetooth. In support, we provide an extensive study of bone conduction characteristics and modulation schemes for digital data communication based on OsteoConduct. We present prototype designs and user studies for the applications of OsteoConduct in both body-area data communication and interfacing. Our experimental results demonstrate that mechanical stimuli can be reliably transmitted through the human musculoskeletal system with power consumption of multiple $\mathrm{mW}$. We also show that excitations generated by human teeth clacks can be readily employed by users to interact with computers and body-area devices. The key components of our OsteoConduct prototypes are a low-power mechanical stimulator, sensor-based receivers, and signal processing techniques for robust data transmission.
\end{abstract}

Categories and Subject Descriptors: C.2.m [Computer systems organization]: Computer-Communication Networks---Miscellaneous; D.2.2 [Software Engineering]: Design Tools and Techniques---User interfaces

General Terms: Design, Measurement, Human Factors.

Keywords: Bone conduction, Body-area network, Personal-area network.

\section{INTRODUCTION}

Radio frequency (RF) technologies dominate wireless body-area communication. For example, Bluetooth has become increasingly available on mobile personal devices, such as cell phones. To reduce the power cost, Wibree, an ultra-low power and low duty cycle radio technology for wireless body-area communication has been recently announced. Nevertheless, body-area communication based on $\mathrm{RF}$ technologies suffers in security and reliability due to the use of the free space for radio propagation.

Permission to make digital or hard copies of all or part of this work for personal or classroom use is granted without fee provided that copies are not made or distributed for profit or commercial advantage and that copies bear this notice and the full citation on the first page. To copy otherwise, to republish, to post on servers or to redistribute to lists, requires prior specific permission and/or a fee.

BODYNETS 2007, June 11-13, Florence, Italy

Copyright $\odot 2007$ ICST 978-963-06-2193-9

DOI 10.4108/bodynets.2007.18
In this work, we propose a novel technology, called OsteoConduct. OsteoConduct enables wireless body-area communication based on mechanically excited bone conduction inside the human musculoskeletal system. We intend it as a secure, reliable, low-power, low cost, and low-data rate alternative to existing RF technologies. An OsteoConduct system includes a stimulator that excites the human bones and a receiver that detects the vibration in the bone. The stimulation can be either user-produced, e.g., through teeth clacks and finger snaps, or externally inflicted, e.g., through a low-power vibrator. Our measurements and theoretical analysis showed that ultra low-power (below $1 \mathrm{~mW}$ ) excitation is enough for fairly reliable communication $(<10 \%$ bit error rate), without being noticeable to the user. The receiver can be a low-cost microphone or accelerometer, thus extremely powerefficient. Our initial investigation and experimental results have shown that OsteoConduct has the potential to interconnect body-worn or implanted devices and provide users with alternative ways to interact with them.

In particular, OsteoConduct is free of radiation and requires extremely low power to maintain a connection and transfer data. It can be ideal for the following application scenarios.

- Exchange low-data rate information with implanted devices;

- Interact with body-worn devices in a hand-free fashion, e.g., to answer a phone call through the Bluetooth headset by a teeth clack, as we will demonstrate in Section 5;

- Manage a power-hungry RF wireless body-area connection as a secondary ultra-low power channel, or wake-on-vibration [11]. For example, Bluetooth connection between a headset and a cell phone can be shutdown between calls and be reestablished upon a request from the cell phone through OsteoConduct; and

- Body-area data communication in a hostile environment, where radio frequencies are likely to be jammed or insecure. 
To the best of our knowledge, OsteoConduct is the first reported body-area communication technology based on the human musculoskeletal system. Our contributions include

- We have conducted extensive measurement to study the bone conduction channel for digital communication and its modulation methods;

- We have prototyped OsteoConduct stimulators and receivers for wireless body-area data communication. Our experimental results demonstrated that the lowrate data can be reliably exchanged between two devices worn at different body anatomic locations; and

- We have designed and implemented a body-area user input method, TeethClick, based on OsteoConduct. TeethClick employs a low-cost microphone to detect deliberate teeth clacks for user input in a hand-free fashion.

It is important to note that OsteoConduct is still an emerging technology. We believe its performance and power consumption will be significantly improved after further research investment.

The rest of the paper is organized as follows. In Section 2, we provide necessary background information regarding the use of bone conduction for body-area communication. We also discuss non-RF body-area communication technologies related to OsteoConduct. In Section 3, we present the system design of OsteoConduct. In Section 4, we offer our experimental measurement of bone conduction channel and explore the use of OsteoConduct for body-area digital data communication. In Section 5, we present TeethClick, a hand-free user input method based on OsteoConduct. We also provide results from our user studies. We discuss the limitations of OsteoConduct and future work in Section 6.

\section{BACKGROUND}

Before presenting our design and implementation of the bone-conduction based body-area communication and interfacing, we next provide background information regarding the nature of bone conduction and discuss related work.

\subsection{Bone Conduction}

Acoustic sound wave propagation through bone tissue is a widely-used technique in evaluating bone properties, specifically determining bone properties through speed-ofsound measurements. This is particularly critical for diagnosing and monitoring the progression of osteoporosis as well as assessing the extent of fracture healing in long bones $[8,16]$. Such systems measured speed of sound and/or broadband attenuation coefficient and correlate these parameters to bone properties. Nevertheless, these devices operate in the ultrasound range with very limited penetration depth into hard connective tissues such as bones. Reliable sound wave propagation using ultrasound is only possible within a few millimeters.

Recent studies have also suggested the applicability of bone-conducted sound waves in hearing aids for the severely disabled [9]. Acoustic wave bone conduction hearing aids have been found capable of supporting frequency discrimination and speech detection for hearing impaired and profoundly deaf subjects [18]. These systems use analog acoustic signals within the audible range and are placed at the outer ear. The skull is used to propagate the sound waves from the outer ear to the inner ear without modification of the analog signal.

Reliable acoustic wave propagation, however, can only be achieved in the low-frequency range, typically around the resonance frequency of the tissue. Resonance of the tissue has the advantage that the whole tissue is excited, instead of a few millimeters as with ultrasound. The downside is the low data transfer rate if serial data communication is applied.

\subsection{Related Work}

Several non-RF body-area communication techniques have been proposed. Microsoft patented a technique that employs skin-conductivity to transmit power and data to body-worn devices [17]. Sun et al. [15] patented a technique that employs current pulses to transmit data to an implanted device through human body. Both techniques suffer from an extremely limited range, due to the low and uneven electrical conductivity of the human body.

Numerous works exist on the vibration characteristics of various human bones, especially human skulls for the application in hearing aid [12-14]. However, none of them considered the use of bone conduction for body-area communication and interfacing. Fukumoto designed and implemented a finger-ring shaped handset [2] to deliver voice through bone conduction of a finger, of which the tip is inserted into the ear. While it is the most related work to ours, it transmitted analog signal through an extremely short range. It is targeted at noise suppressing instead of body-area communication.

\section{SYSTEM DESIGN OF OsteoConduct}

As illustrated in Figure 1, an OsteoConduct system consists of three components: a stimulator that produces mechanical excitations, part of the human musculoskeletal system that conducts the vibrations, and a receiver that detects the vibration.

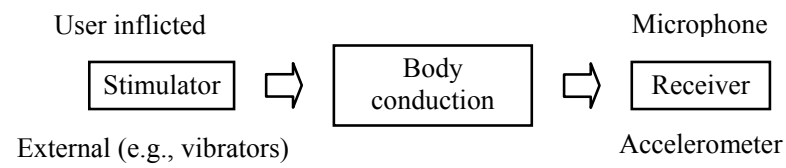

Figure 1. System view of OsteoConduct 
Human users can easily produce bone vibrations, e.g., through teeth clacks and finger snaps. Such user inflicted excitation can be readily used for interfacing body-area devices. In Section 5, we will present an input method based on detecting bone-conduction excited by deliberate gentle teeth clacks.

Bone-conduction can also be excited externally. For example, vibrators on mobile phones can be used to generate frequency patterns in the upper audible range. Low-frequency vibration patterns are commonly generated by either vibration motors or electromagnetic shakers. In vibration motors, the amplitude and frequency are coupled through a mechanical link to the eccentric weight. Increasing the motor speed will also increase the excitation. Electromagnetic shakers do allow for a separation between amplitude and frequency. Through power limiting components a flat power spectrum of the shaker can be achieved, allowing robust data communication between different devices at alternate frequency ranges.

For our experiments, we have built a reaction-type lowpower electromagnetic shaker to generate dynamic forces. In essence, it works in a similar way as a louder speaker but in different frequency range. This type of shakers offers a lightweight and compact configuration, ideal for miniaturization. In addition, such shakers are designed for operation over a very wide range of audio frequencies. In our experiments described below, the power consumption of the shaker is measured between 0.4 and $1 \mathrm{mw}$.

Bone-conduction can be detected using microphones or accelerometers with coupled amplifiers. Both consume minuscule power. Since microphones are generally less sensitive, we employ sensitive accelerometers for digital data communication (Section 4) and employ microphones for detecting the existence of bone-conduction (Section 5).

\section{DATA COMMUNICATION}

We next present our experimental results regarding the use of OsteoConduct for body-area digital data communication. We first describe our test platform, then provide our findings regarding modulation schemes and properties of the communication channel, and finally present our prototype implementations.

\subsection{Experimental Setup}

The electromagnetic shaker described above is employed to produce bone conduction. An ultra low-power MEMSbased three-axis accelerometer from Kionix [6] is held against the receiving body location as the receiver. A LabVIEW program controls the entire system. It generates the input sequence in sinusoids with different modulation to drive the electromagnetic shaker. The same program receives the signal from the accelerometer and demodulates the signal. The received bit sequence is then compared to the input sequence to calculate accuracy.
For the first set of measurements, we focus on the forearm bone. The arm of a subject is placed on the armrest of the device and strapped into the wrist holder. Our LabVIEW software controls the input voltage to the electromagnetic shaker to produce vibrations into the wrist, with different gravitational force (g's). The bone conduction is measured at the elbow joint by an accelerometer.

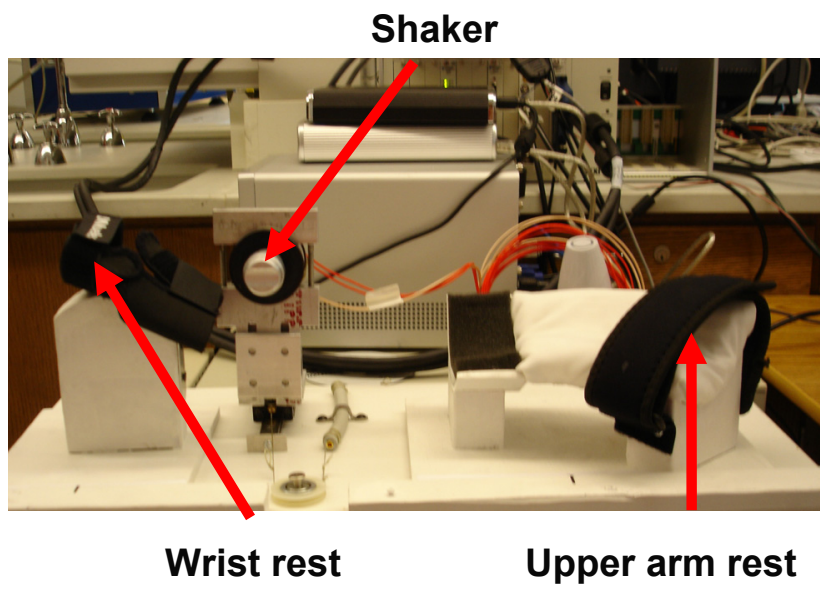

(a) Test platform with the electromagnetic shaker

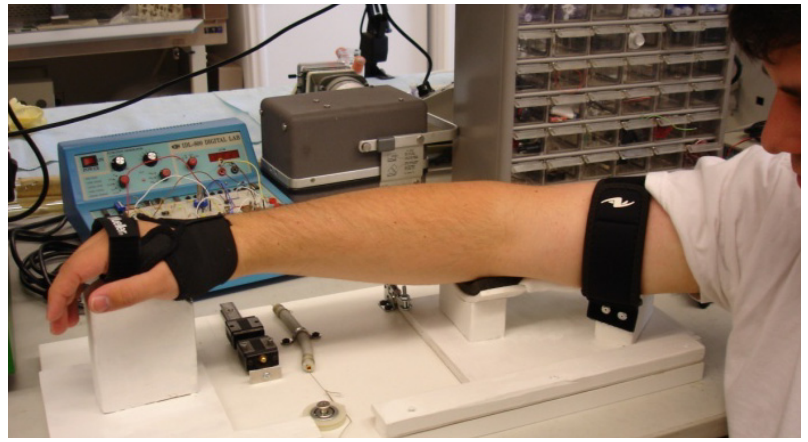

(b) Test platform with a subject's forearm in (Shaker was not present)

Figure 2. Test platform

\subsection{Modulation Schemes}

We have examined ASK (amplitude shift-keying) and FSK (frequency shift-keying) for OsteoConduct data communication, primarily due to their simplicity. We have developed a LabVIEW program to encode the raw bits into modulated signals to control the input voltage of the electromagnetic shaker, as described above.

In FSK modulation, on and off frequencies are chosen for constant amplitude. The range of on frequencies used was between the lower shaker bound of $10 \mathrm{~Hz}$ and a selected upper bound of $2000 \mathrm{~Hz}$, with the off frequency being defined in terms of the on frequency interval. The receiver determines the frequency using LabVIEW's built-in 
Buneman frequency estimator. For ASK modulation, the input signal frequency is held constant, while different amplitude values are assigned to a chosen bit pattern. We have employed 0-0.001g for the off amplitude and 0.1-1.0g for the on amplitude. Follows are our important findings.

Firstly, FSK consistently outperform ASK in accuracy when similar vibration forces are employed. ASK suffers more from the muscle attenuation and changes in muscular contraction. The demodulator of FSK is much simpler and more robust than that of ASK. Therefore, we focus on the properties and performance of FSK in the follows.

Secondly, $300-350 \mathrm{~Hz}$ is best for FSK for the arm bone examined. We performed frequency sweeps over the range of $10-2000 \mathrm{~Hz}$. $300-350 \mathrm{~Hz}$ demonstrated the least amplitude attenuation, as showed in Figure 3. Our later experiments showed this frequency range also works well for other part of the skeleton system and for other subjects.

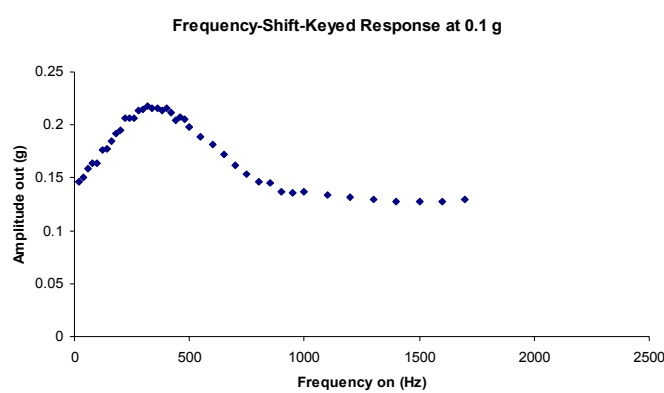

Figure 3. Frequency-Shift-Keyed response at 0.1g. Performing a frequency sweep at $0.1 \mathrm{~g}$ has revealed that the optimal transmission frequency for the ulna for this particular subject is between $300-350 \mathrm{~Hz}$

Thirdly, we found that muscular contraction pushes the best frequency range toward the higher end slightly, because of the increase in the effective resonant frequency of the channel (bone and muscle). These results are similar to those reported by Jurist in 1970 [3]. The effect must be taken into consideration for any potential future in vivo applications of the experimental system. Therefore, the FSK on and off frequencies should be separately by a wide range so that muscular contraction will not reduce the receiving accuracy.

\subsection{Communication Accuracy and Data Rate}

We next present the results from our second set of experiments regarding the communication accuracy and data rates of OsteoConduct. Two subjects, one male and one female, participated in our experiments. Both subjects were in their mid-twenties and of medium build. The trials were run while both subjects were standing straight up.

FSK is employed and the electromagnetic shaker is driven by $100 \mathrm{mV}$, with power consumption below $1 \mathrm{~mW}$. The off and on frequencies are 350 and $320 \mathrm{~Hz}$, respectively. At such low amplitude, the vibration is not perceptible to the subjects.. A 2048 bit random sequence is used as the test signal. The transmission rate is almost $5 \mathrm{bits} / \mathrm{sec}$. The signal is applied to the body by placing the piezo shaker in contact with the designated body part. Figure 4 shows the setup. Two different transmitter locations were tested: the wrist (1) and the lower back (2). The setup of Figure 2 is used for transmitting from the wrist. For transmitting from the lower back, the electromagnetic shaker is taken off from the platform and held to the lower back. Three different receiver locations were tested: the wrist (1), the lower back (2), and behind the ear (3). We select these three locations because socially accepted devices have been worn at these locations: wrist for watches, lower back for belts and cell phones, and behind the ear for headsets.

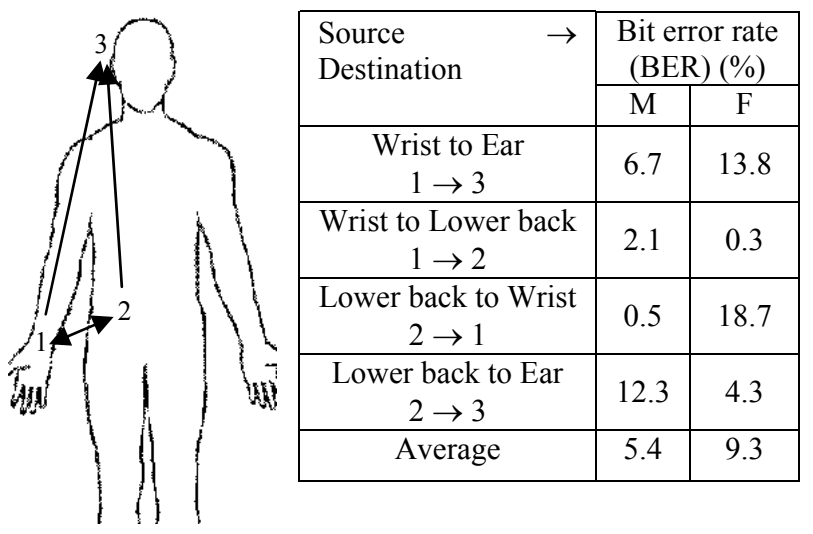

Figure 4. Experimental Setup: Tests were ran for transmitter locations of wrist (1) and lower back (2) and receiver locations of wrist (1), lower back (2), and behind the ear (3)

Figure 4 summarizes our measurement results. First of all, OsteoConduct achieves $<10 \%$ bit error rate without any error correction. It is quite amazing because all four links involve multiple bones and many joints. Second, the performance can be asymmetric. For example, the female subject has much lower BER from the wrist to the lower back than from the lower back to the wrist. Thirdly, the difference between subjects is considerable. On average the male subject enjoys a much lower BER. One possible reason is males in general have lower body-fat percentage and fat attenuates the bone conduction more than muscles.

\subsection{Stimulator and Receiver Prototypes}

We have built an ultra-low power receiver in the form factor of a wrist-watch, which is shown in Figure 5. It employs the same ultra-low power three-axis accelerometer used in the experiments and an ultra-low power microcontroller (MSP430) from Texas Instruments. The active power consumption during OsteoConduct receiving is below $5 \mathrm{~mW}$. We are currently investigating the use of the built-in vibrators in cell phones as the simulator. 


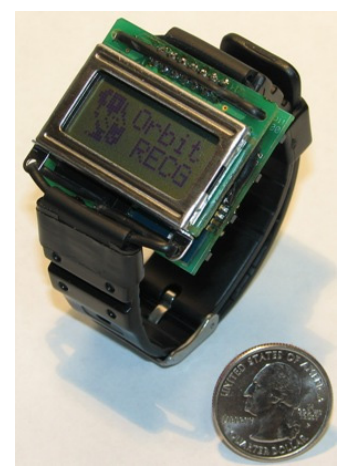

Three-axis accelerometer picks up bone vibration

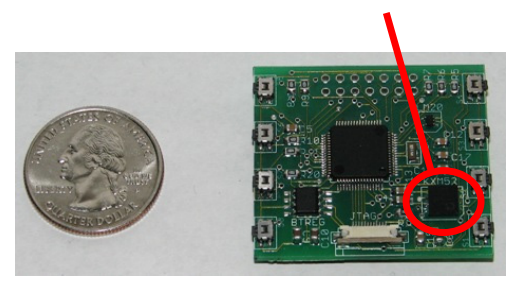

Figure 5. Digital wrist watch with a three-axis accelerometer as the OsteoConduct receiver

\section{USER INTERFACE}

We next present our implementation of a hand-free user input method, called TeethClick, using gentle yet deliberate teeth clacks based on OsteoConduct. TeethClick employs a low-cost throat microphone to pick up bone-conduction signal from the cheek. It uses very efficient spectrum analysis to distinguish teeth clacks from other vocal activities. Our implementation and analysis are based on the throat microphone from a Noise Terminator headset from IASUS Concepts [1].

\subsection{Bone-Conduction Signal of Teeth Clacks}

The bone-conduction signal of teeth clacks is characterized by high energy in spectrum above $2000 \mathrm{~Hz}$ but low energy below it. Figure 6 shows the time-spectrum of the boneconduction signal of several teeth clacks. The spectrum of the bone-conduction signal of speech, as shown in Figure 7, is almost the opposite. It is characterized by high energy in spectrum below $2000 \mathrm{~Hz}$ but low energy above it. This dramatic difference is introduced by that the skin and skull is a much lower-pass filer to acoustic signals than to bonevibration incurred by teeth clacks. This forms the basis for our algorithm to detect teeth clacks.

\subsection{Detecting Deliberate Teeth Clacks}

For low-power and real-time implementation, we design a simple-yet-effective algorithm based on the property of the bone-conduction signal. The algorithm simply examines the energy densities in the lower and higher spectral ranges of the bone-conduction signal. High energy density in the lower spectral range indicates the existence of speech, while a sudden increase in the energy density in the higher spectral range indicates the occurrence of a teeth clack. A deliberate teeth clack is detected if a teeth clack occurs without the presence of speech.

Our implementation is based on standard speech signal processing: We sample the bone-conduction signal and divide the samples into overlapping frames. In our implementation, each frame is about $23.3 \mathrm{~ms}$ and adjacent frames are about $22 \mathrm{~ms}$ apart. For each frame, we conduct FFT to get its spectrum. In our implementation, the "low" spectral range is between 0 and $2750 \mathrm{~Hz}$, while the "high" spectral range is between 1875 and $5500 \mathrm{~Hz}$. Through experiments, we discovered that such overlapping ranges work best. For the $n$th frame, we calculate the energy densities in the low and high spectral ranges, denoted as $A_{n}$ and $B_{n}$, respectively. We also keep a record of the average energy density of silence, $U$.

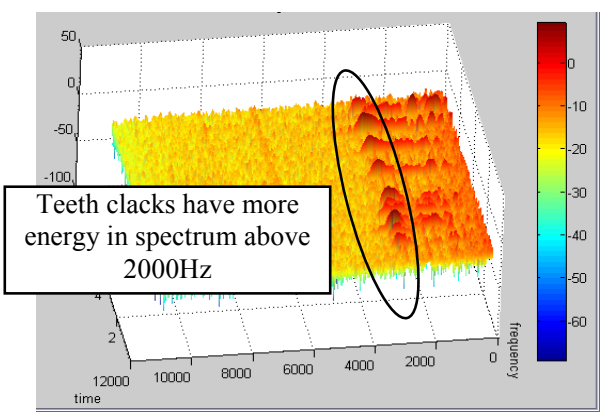

Figure 6. Spectrum vs. time for bone-conduction signal of a series of deliberate teeth clacks

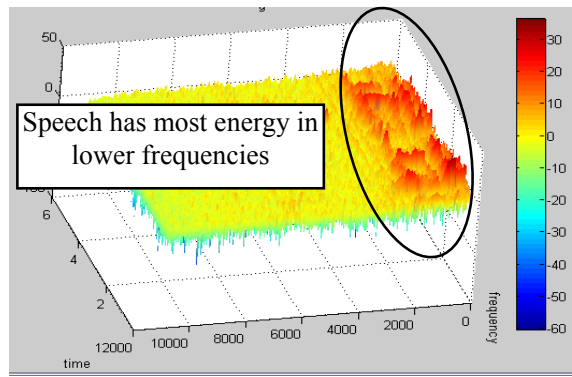

Figure 7. Spectrum vs. time for bone-conduction signal of speech

If $B_{n}$ is considerably larger than $B_{n-1}$ and $B_{n+1}$, the algorithm declares that a teeth clack is detected. For accidental teeth clacks, $A_{n-1}$ and $A_{n+1}$ are large due to the presence of speech. Therefore, the algorithm declares that a deliberate teeth clack is detected if and only if $B_{n}$ is considerably larger than $B_{n-1}$ and $B_{n+1}$ AND $A_{n-1}$ and $A_{n+1}$ is on the same level as the $U$. Let $C_{n}$ be the Boolean logic that evaluates whether a deliberate teeth clack is detected for the nth frame. It can be formulated as

$$
\begin{gathered}
C_{n}=\left[\left(B_{n-1}+\text { offset }\right)<B_{n}\right] \text { and }\left[\left(B_{n+1}+\text { offset }\right)<B_{n}\right] \\
\text { and }\left[A_{n-1} \leq(U+\text { offset })\right] \text { and }\left[A_{n+1} \leq(U+\text { offset })\right]
\end{gathered}
$$

where offset is empirically set to $5 \mathrm{~dB}$. It is important to note that while the algorithm is based on the generic property of the bone-conduction signal, its implementation is highly dependent on the property of the throat microphone. In our implementation, the low and high spectral ranges as well as the offset were empirically determined by examining the bone-conduction spectrum.

\subsection{Input with Teeth Clacks}

If we view a teeth clack as a tact button push, we can have different inputs for consecutive multiple clacks. To distinguish between a "single click" and a "double click", 
we continuously analyze a first-in first-out (FIFO) buffer that stores $C_{n}$ for the most recent frames within $400 \mathrm{~ms}$. If a teeth clack is detected and no teeth clack is detected in following $300 \mathrm{~ms}$, we treat it as a single click. If two consecutive teeth clacks are separated by less than $100 \mathrm{~ms}$, we treat them as the one clack and use earlier time as its on-set time. If two consecutive clacks are separated by more than $100 \mathrm{~ms}$ and less than $300 \mathrm{~ms}$, we treat them as a double click. The rate of single clicks is limited by the $300 \mathrm{~ms}$ delay for every single click that we have to wait to tell whether it is part of a double click.

Single clicks are faster and require less effort than doubleclicks; they should be used for more frequent tasks. This is similar to the philosophy of Huffman coding, which uses shorter code words for more frequent symbols.

Our tests showed that this algorithm provides reliable detection of single and double clicks. Extending it to triple clacks will require user-specific calibration and introduces further latencies in single-click detection. Therefore, TeethClick only uses single and double clicks, effective implementing a tact button with teeth clacks.

Teeth clack location: We considered recognizing clacks at different locations of the jaw, using two throat microphones placed on both sides of the cheek. We found that recognizing the location is difficult and unreliable, because it requires stereo processing and multiple microphones. It is very sensitive to the positions of microphones. Moreover, producing located teeth clacks requires much higher physical effort and can easily introduce facial muscle strain. Therefore, we choose not to use teeth clack location.

\subsubsection{User interface with TeethClick}

TeethClick is functionally the same as a mouse button. We intend it as an auxiliary input technique to other more versatile input technologies. However, TeethClick enjoys the advantage as being hand-free and non-intrusive. By carefully designing the user interface, TeethClick can be used by people with motor impairments. In this Section, we present two examples that implement two basic GUI operations: selection and pointing. Selection refers to choose one item from a list. Pointing refers to move the cursor to a certain position inside a window.

Selection: To select from a list of items, a single click highlights the next item, which is a more common and often repeated task. A double click selects the current highlighted item.

Pointing: We can build most GUI operations based on the selection operation. A user can use TeethClick to move the cursor to any block with two actions, "Rotate" and "Move". "Rotate" changes the cursor orientation. "Move" moves the cursor to the next block along the direction it is oriented. Since "Move" is more frequent than "Rotate", we could use single clicks to "Move" and double clicks to "Rotate." Since "Move" is highly repeated, we use a double click to switch between "Move" and "Rotate" and a single click to repeat the chosen action.

\subsection{User studies}

We implemented TeethClick using the MATLAB data acquisition toolbox. Four subjects, two male and two female ECE graduate students, participated in user studies for the selection and pointing operations with TeethClick. We explained TeethClick as well as the selection and pointing operations to subjects at the beginning. We then allowed them five minutes to play with selection and pointing each. Then we started measuring their performance throughout multiple trials to see the learning curve in a short time.

The task is to use TeethClick to select numbered items from a menu in an arbitrary but predefined sequence. The menu includes only a sequence of numbers, from 1 to 6 , to minimize distraction, as shown in Figure 8. The subject was asked to select the numbers in the order of $3,5,6,4,1$, 5,2 . This task was carried three times with five-minute break in between. The total time to finish each trial is shown in Figure 9. The user performance in the second trial was significantly better than the first one. The primary reason was that subjects made much fewer errors in the second trial. However, the performance slightly deteriorated in the third trial. According to our post experiment interviews, this was due to that the subjects became overconfident after two trials when they realized how easy TeethClick was and became less focused.

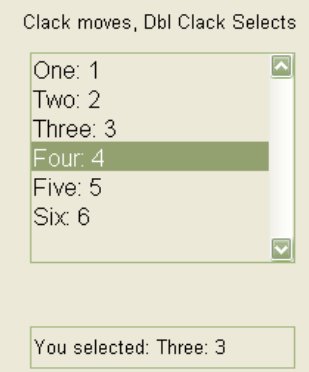

Figure 8. Selection with single-clack to move and double-clack to select

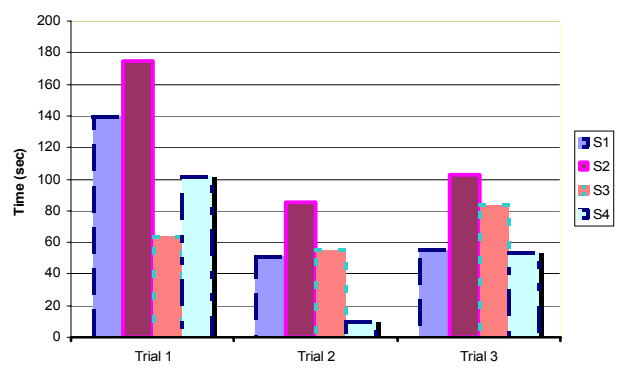

Figure 9. Performance for the selection task

We also conducted user studies with the pointing task. More details and a video demo can be found at [7]. 


\subsubsection{Subjective Evaluations}

After a subject finished the experiments, we asked her/him to fill a questionnaire for a subjective evaluation. We also interviewed the subjects for questions we had with their behavior in the experiments and their answers in the questionnaire. We summarize our findings as follows.

We asked subjects how comfortable they feel with using TeethClick in the experiments. The average level of comfort is 3.5 with 1 being "Extremely uncomfortable" and 7 being "No problem at all". There was a concern of jaw strain after elongated usage. This concern was unexpected because TeethClick requires very gentle teeth clacks with little facial muscle effort. However, we observed that all our subjects made strenuous teeth clacks in the experiments, which introduced discomfort after 45 minutes of TeethClick usage, despite that we told them gentle clacks work as well. We found out from interviews that they unconsciously assumed that a forceful clack could be more accurate. They also felt in better control when forceful clacks were used. Since it was the first time our subjects had used TeethClick and they had only about 45 minutes of experience with it, we believe these issues will become less a problem after enough exposure to TeethClick. However, a more extensive and longer term user study is necessary to investigate them. Our subjects indicated a 6.5 average level of comfort for wearing the throat microphone, based on the same 1-7 scale.

The average subject satisfaction score for TeethClick speed is 4.25 , as 1 being "Very disappointed" and 7 being "Very satisfied". Our subjects also noticed that the GUIs used in experiments can be improved in many aspects for higher performance. This highlights the importance in designing user interfaces based on TeethClick. Another note is that our measurement showed that users can easily make 4 to 5 teeth clacks per second, a speed comparable to mouse button clicks. This indicates that TeethClick has a lot of room for improvement.

To summarize, TeethClick is a new technique to users and many issues remain to be addressed. The most important issue is to ensure user confidence in using gentle teeth clacks. Our subjects did recognize that TeethClick has its own value and recommend it for people who need handfree computer operations.

\subsection{Related Work in Hand-Free Input}

Input techniques similar to TeethClick were explored in the past, especially for people with motor impairments. Some used the tongue and in-mouth springs, switches, or joysticks. For example, Kingma and Sabourin developed a "mouth-mouse" for quadriplegic computer users [5]. A mouth-mouse user uses the tongue to push several in-mouth springs for mouse moving and bites a switch for mouse clicking. Similar tongue-operated in-mouth input devices were reported in $[4,10]$. TeethClick is much more hygienic, less intrusive, and easier to operate. Since
TeethClick uses the bone-conduction signal, it is almost immune from environmental noise.

\section{DISCUSSIONS AND CONCLUSIONS}

We presented a novel body-area wireless communication technology based on bone-conduction. We conducted an extensive study of bone conduction characteristics and proper modulation schemes for low-power reliable digital data communication through the human musculoskeletal system. We chose FSK due to its simplicity and resilience against the attenuation from soft tissues. Our experiments showed that data communication at 5 bits/sec can be achieved between body locations far apart (wrist, lower back, and behind ear) with an average bit error rate below $10 \%$ without any error correction. The results showed the potential of OsteoConduct as a low-power low data-rate alternative to existing RF-based wireless body-area communication technologies. We have built an ultra-low power receiver prototype in the form factors of a wrist watch. We are currently investigating the use of the built-in vibrator of commercial cell phones for the stimulator. We presented the application of OsteoConduct to body-area user interface, TeethClick, which employs teeth clacks to produce user inputs. Combined with proper user interface designs, TeethClick can be used to operate computers hand-freely. Our user studies showed that TeethClick is easy to learn and achieve decent performance even in GUImouse tasks, such as selection and cursor pointing, making it an ideal low-cost complementary/auxiliary technique to speech recognition.

OsteoConduct is an emerging technology. The work presented in this paper is our first step toward making it a reliable, low-power, and secure alternative to existing RFbased wireless technologies for body-area communication, which have been extensively researched for decades. While OsteoConduct is still in its nascent form, our results have shown its potential.

Our ongoing works are aimed at achieving such potential and evaluating OsteoConduct through field studies with prototype implementations. In particular, we are working on

- Improving data communication accuracy through efficient error correction;

- Improving data communication rate through advanced modulation, especially multiple frequency-shift keying and tradeoffs between accuracy and raw data rate;

- Prototype implementation of an OsteoConduct stimulator using vibrators common to cell phones and in field evaluation of OsteoConduct data communication from a cell phone belt worn to the wrist-worn receiver described in Section 4.4;

- Extensive characterization of OsteoConduct channels of candidate applications, in particular, the four links 
measured in Section 4.3, throughout users' daily activities; and

\section{- Health impact of OsteoConduct}

While promising, OsteoConduct is limited in the following aspects, compared with RF-based technologies, for bodyarea data communication. First of all, its data rate will be extremely limited, due to the nature of the human skeleton system. Second, the performance of an OsteoConduct system is likely to be highly dependent on the physical condition of the user. It will be extremely difficult to provide a system that works well for all users. Third, for body-worn devices, OsteoConduct requires them to touch the human body. Such requirements may be difficult to satisfy for some devices, especially during physical activities. In these scenarios, OsteoConduct-based data communication can become opportunistic and technologies from disruption-tolerant network should be employed. Fourth, although OsteoConduct incurs extremely low power consumption, the energy per bit transferred may not be low, due to its extremely low data rate. Therefore, OsteoConduct may not be suitable for data intensive applications.

Despite of these limitations, we believe OsteoConduct can be a complementary/alternative technology to RF-based wireless body-area communication when security and lower power consumption becomes critical and a low-data rate suffices. More importantly, the nature of OsteoConduct makes it an ideal nature for simple user interactions with body-worn or implanted devices, as we have demonstrated in this work.

\section{ACKNOWLEDGEMENTS}

This work was made possible in part through support from Texas Instruments Leadership University Innovation Fund. The authors would like to thank all the anonymous volunteers who participated in our user studies and experiments.

\section{REFERENCES}

[1] IASUS Concepts: Noise Terminator (NT) http://www.iasusconcepts.com/noise terminator/.

[2] Fukumoto, M., A finger-ring shaped wearable handset based on bone-conduction. in Proceedings of IEEE International Symposium on Wearable Computers, (2005), 10-13.

[3] Jurist, J.R.I. In vivo determination of the elastic response of bone: I. Ulnar resonant frequency in osteoporotic, diabetic and normal subjects. PHYS. MED. BIOL, 15 (3). 427-434.

[4] Kim, D., Tyler, M.E. and Beebe, D.J. Development of a tongue-operated switch array as an alternative input device. International Journal of Human-Computer Interaction, 18
(1). 19-38.

[5] Kingma, Y.J. and Sabourin, P.J., Development of a mouthmouse for quadriplegics. in Proceedings of the Annual International Conference of the IEEE Engineering in Medicine and Biology Society, (1989), 1516.

[6] Kionix www.kionix.com.

[7] Mohamed, T. and Zhong, L. TeethClick: Technical report and video demo. http://www.ruf.rice.edu/ mobile/publications/teethclick06mo hamed.pdf and http://www.ruf.rice.edu/ mobile/publications/clackdemo.wm $\underline{v}$.

[8] Nicholson, P.H.F., Moilanen, P., Karkkainen, T., Timonen, J. and Cheng, S. Guided ultrasonic waves in long bones: modelling, experiment and in vivo application. Physiol. Meas, 23 (4). 755-768.

[9] Sakaguchi, T., Hirano, T., Watanabe, Y., Nishimura, T., Hosoi, H., Imaizumi, S., Nakagawa, S. and Tonoike, M. Inner head acoustic field for bone-conducted sound calculated by finite-difference time-domain method. Jpn. J. Appl. Phys, 41. 3604-3608.

[10] Salem, C. and Zhai, S. An isometric tongue pointing device Proceedings of the SIGCHI Conference on Human Factors in Computing Systems (CHI), Atlanta, GA, 1997.

[11] Shih, E., Bahl, P. and Sinclair, M.J. Wake on wireless: An event driven energy saving strategy for battery operated devices. Proceedings of ACM Annual International Conference on Mobile Computing and Networking (MobiCom).

[12] Sohmer, H., Freeman, S., Geal-Dor, M., Adelman, C. and Savion, I. Bone conduction experiments in humans-a fluid pathway from bone to ear. Hear Res, 146 (1-2). 81-88.

[13] Stenfelt, S. and Goode, R.L. Transmission properties of bone conducted sound: measurements in cadaver heads. $J$ Acoust Soc Am, 118 (4). 2373-2391.

[14] Stenfelt, S., Håkansson, B. and Tjellström, A. Vibration characteristics of bone conducted sound in vitro. The Journal of the Acoustical Society of America, 107. 422.

[15] Sun, M., Sclabassi, R.J. and Mickle, M.H. Method of data communication with implanted device and associated apparatus. Patent, U.S. ed., University of Pittsburgh of the Commonwealth System of Higher Education, Pittsburgh, PA, USA, 2005, 14.

[16] Tatarinov, A., Sarvazyan, N. and Sarvazyan, A. Use of multiple acoustic wave modes for assessment of long bones: Model study. Ultrasonics, 43 (8). 672-680.

[17] Williams, L., Vablais, W. and Bathiche, S.N. Method and apparatus for transmitting power and data using the human body. Patent, U.S. ed., Microsoft Corporation, Redmond, WA, USA, 2004, 15.

[18] Yang, D., Xu, B., Wang, X. and Jia, X., The study of digital ultrasonic bone conduction hearing device. in Proceedings of the Annual International Conference of the IEEE Engineering in Medicine and Biology Society, (2005), 18931896. 